\title{
Frequency of Using Miniscrew Anchorage by the Iranian Orthodontists
}

\author{
Amin Golshah ${ }^{1}$, Mohadese Feiz Rahnemoon ${ }^{2 \star}$, Zahra Bagheri ${ }^{2}$, Nasim Karami ${ }^{2}$ \\ ${ }^{1}$ Department of Orthodontic, School of Dentistry, Kermanshah University of Medical Sciences, Kermanshah, Iran; ${ }^{2}$ Students \\ Research Committee, School of Dentistry, Kermanshah University of Medical Sciences, Kermanshah, Iran
}

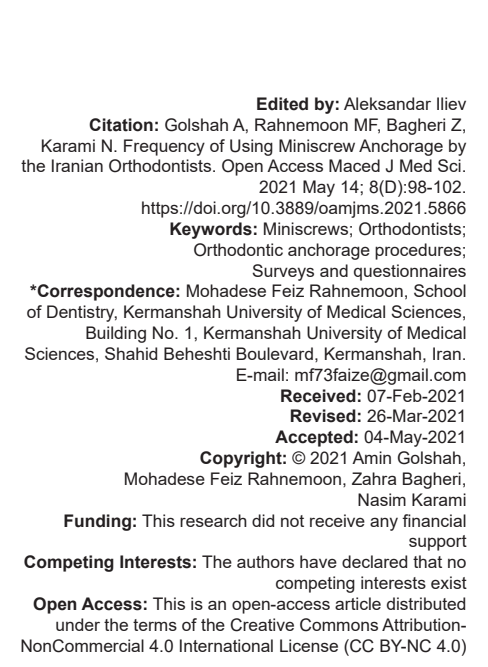

\begin{abstract}
AIM: In recent years, miniscrews are extensively used for anchorage in orthodontic treatments. This study aimed to assess the frequency of using miniscrew anchorage by the Iranian orthodontists in 2020.

MATERIALS AND METHODS: This descriptive, cross-sectional study evaluated 70 Iranian orthodontists. After obtaining their written informed consent, a questionnaire was emailed to them. The validity of the questionnaire was confirmed by four faculty members and its reliability was determined to be $75 \%$ by calculating the Cronbach's alpha after administering it among 40 orthodontists. Data were analyzed using SPSS version 22.

RESULTS: Of 97 administered questionnaires, 70 were filled out and returned (response rate=72.16\%) by 42 males and 28 females. Of participants, $62.3 \%$ reported using miniscrews in their practice. Furthermore, $96.6 \%$ of the orthodontists reported that they would place the miniscrews by themselves. The majority of orthodontists $(98.3 \%)$ were completely or relatively satisfied with the efficacy of miniscrews, and $93.5 \%$ recommended the use of miniscrews to their colleagues. Factors such as poor expertise in miniscrew insertion, doubts about its advantages, high cost, and time-consuming placement were the main reasons for reluctance of some orthodontists in using miniscrews.

CONCLUSION: Considering the high efficacy of miniscrews for orthodontic treatment and high satisfaction level of Iranian orthodontists with miniscrews, their application is expected to rise by the Iranian orthodontists after eliminating the existing limitations against their widespread use.
\end{abstract}

\section{Introduction}

Anchorage is an important requirement in orthodontics. Several tools and strategies may be used for this purpose, including the use of external anchorage devices such as headgears and intraoral devices such as the Nance appliance or the patient's teeth. Although such anchorage devices can somehow serve the purpose, unwanted tooth movements are inevitable [1], [2]. Thus, a more efficient and safe tool/ strategy is required for this purpose.

In recent years, miniscrews have gained increasing popularity as anchorage in orthodontic treatments. Miniscrews are conical-shaped small screws made of titanium. They are temporarily inserted in the cortical plate of the maxilla or mandible. The miniscrew head is connected to the teeth to cause tooth movement toward the desired position [3]. The other advantages of miniscrews include relatively low cost, easy application, and insertion [4]. The miniscrews can be used for orthodontic treatment by two methods of self-tapping and self-drilling [5].
On the other hand, loosening is a shortcoming of miniscrews. However, its occurrence can be minimized by selecting a miniscrew with optimal length and diameter and also by appropriate selection of the site and angle of miniscrew placement. Some conditions such as impaired healing, immunodeficiency, hematological disorders, poor bone quality, and poor oral hygiene limit the application of miniscrews as well [4]. The frequency of usage of miniscrews varies in different countries worldwide. A previous study evaluated 1691 orthodontists in India and reported that $56.3 \%$ had never used miniscrews while $43.7 \%$ reported the use of miniscrews for their patients [6]. Of French orthodontists, $66 \%$ reported the use of miniscrews in their daily practice [7]. In the United States, miniscrews are less commonly used, although orthodontists favored miniscrews in five states [8].

Considering the fact that use of miniscrews requires a high level of expertise acquired by participation in workshops and educational programs as well as adequate experience and tools, and reluctance of some orthodontists to use miniscrews due to possible complications during their placement, this study aimed to assess the popularity and frequency of use of 
miniscrews by the Iranian orthodontists to find reasons behind their reluctance and come up with strategies to eliminate them.

\section{Subjects and Methods}

This descriptive, cross-sectional study evaluated 70 Iranian orthodontists. The minimum sample size was calculated to be 60 orthodontists according to a previous study by Barthelemi and Beauval [7], assuming 95\% confidence interval, $80 \%$ study power. For more confidence, 70 orthodontists were requested to participate in this study.

Data were collected using a questionnaire developed by Barthelemi and Beauval [7]. The questionnaire was first translated to Farsi and its questions were naturalized according to the standard method. The validity of the questionnaire was confirmed by four faculty members and its reliability was calculated to be 0.75 by administering it among 40 participants and calculation of Cronbach's alpha using test-retest reliability.

The questionnaire used in this study included four sections of demographic information, miniscrew users, miniscrew non-users, and final questions.

The first section contained 11 questions and asked for the demographic information of orthodontists such as their age, gender, clinical practice history, knowledge level about miniscrews, interest in miniscrew usage, technique of orthodontic treatment, technique of anchorage use, frequency of miniscrew usage, main reason for not using miniscrews, factors that can change the opinion of orthodontists regarding the use of miniscrew, and main factors contributing to future use of miniscrews. All questions in this section were multiple-choice questions and included six questions with four answer choices, three questions with five answer choices, one question with two answer choices, and one last question also with two answer choices.

The second part of the questionnaire included 17 questions regarding miniscrew users: Seven questions with four answer choices, four questions with three answer choices, three questions with five answer choices, two questions with six answer choices, and one question with eight answer choices. The questions in this section asked for the frequency of use of miniscrews, duration of their use, level of satisfaction with their use, target age group for miniscrew insertion, miniscrew placement technique, miniscrew placement site, type of tooth movements performed by use of a miniscrew, strategies to avoid contacting the roots, size of miniscrew (length and diameter) used, type of miniscrew head, brand of miniscrew most commonly used, disinfection method before, during and after miniscrew placement, local anesthesia technique administered before miniscrew insertion, and duration of miniscrew usage in orthodontic treatment.

The third section included three questions for miniscrew non-users including two questions with two answer choices and one question with nine answer choices. The questions asked about the main reasons behind not using miniscrews, and whether or not this decision can be changed, and the factors can contribute to this decision change in the future.

The fourth section included final questions that were asked from all participants. The questions asked about the types of workshops required to enhance the knowledge of clinicians regarding miniscrew usage and whether or not the orthodontists suggest miniscrew usage to their colleagues.

Open questions were not used in this study. Instead, all questions were multiple-choice questions that had been designed such that the acquired scores would indicate the usage rate of miniscrews.

Orthodontists were contacted by email and were requested to participate in the study. The questionnaire and consent form were both emailed to orthodontists. In case of no response, they were contacted again by phone. The questionnaires were filled out anonymously, and the participants were ensured about the confidentiality of their information. Furthermore, participation in the study was voluntary.

Data were analyzed using SPSS version 22. The measures of central dispersion were reported for the quantitative variables, while percentage and frequency values were reported for the qualitative variables.

\section{Results}

Of 97 orthodontists who were invited to participate in the study, 70 returned the questionnaires, including 42 males and 28 females (response rate $=72.16 \%$ ). Table 1 presents the characteristics of the participants. As shown, the majority of participants were between 30 and 39 years $(50 \%)$ and had a clinical practice history of $1-5$ years $(38.6 \%)$ as an orthodontist. Most orthodontists had good knowledge about (65.2\%) and high interest $(62.3 \%)$ in using miniscrews. The buccal technique was the most commonly practiced orthodontic technique (98.6\%), and the most commonly used type of anchorage device was the temporary anchorage device $(62.3 \%)$ by the participants. Furthermore, $89.9 \%$ of the participants reported the current and future use of miniscrews. The $30.2 \%$ of participants emphasized on the need for educational hands-on workshops on the use of miniscrews.

Table 2 shows the parameters related to miniscrew users. Accordingly, $38.6 \%$ of participants 
Table 1: Characteristics of participants

\begin{tabular}{|c|c|c|}
\hline Variables & Frequency & Percentage \\
\hline \multicolumn{3}{|l|}{ Age (years) } \\
\hline $20-29$ & 6 & 8.6 \\
\hline 30-39 & 35 & 50.0 \\
\hline $40-49$ & 23 & 32.9 \\
\hline $50-59$ & 6 & 8.6 \\
\hline$\geq 60$ & 0 & 0 \\
\hline$<1$ & 4 & 5.7 \\
\hline \multicolumn{3}{|l|}{ History of orthodontic practice (years) } \\
\hline $1-5$ & 27 & 38.6 \\
\hline $5-10$ & 17 & 24.3 \\
\hline$>10$ & 22 & 31.4 \\
\hline \multicolumn{3}{|l|}{ Knowledge level about miniscrews } \\
\hline Very good & 18 & 26.1 \\
\hline Good & 45 & 65.2 \\
\hline Poor & 6 & 8.7 \\
\hline Very poor & 0 & 0 \\
\hline \multicolumn{3}{|l|}{ Interest in miniscrew usage } \\
\hline Relatively high & 20 & 29.0 \\
\hline High & 43 & 62.3 \\
\hline Low & 5 & 7.2 \\
\hline None & 1 & 1.4 \\
\hline \multicolumn{3}{|l|}{ Type of anchorage device used } \\
\hline Fixed anchorage auxiliaries & 28 & 40.6 \\
\hline Archwire bends & 18 & 26.1 \\
\hline Extraoral forces (directional forces) & 9 & 13.0 \\
\hline Temporary anchorage devices & 43 & 62.3 \\
\hline Other types & 3 & 4.3 \\
\hline \multicolumn{3}{|l|}{ Most commonly used orthodontic technique } \\
\hline Buccal technique & 68 & 98.6 \\
\hline Lingual technique & 3 & 4.3 \\
\hline Use of clear aligners & 8 & 11.6 \\
\hline \multicolumn{3}{|l|}{ Requirements } \\
\hline Theoretical-clinical workshop & 14 & 22.2 \\
\hline Clinical hands-on workshop & 19 & 30.2 \\
\hline Case presentation workshop & 17 & 27.0 \\
\hline Mechanics workshop & 13 & 20.6 \\
\hline \multicolumn{3}{|l|}{ Frequency of miniscrew usage } \\
\hline Use of miniscrew for patient treatment & 62 & 89.9 \\
\hline Not using miniscrews but planning to use them in the future & 4 & 5.8 \\
\hline $\begin{array}{l}\text { Currently using miniscrews but planning not to use them } \\
\text { anymore }\end{array}$ & 2 & 2.9 \\
\hline Not using miniscrews and no plans to use them in the future & 1 & 1.4 \\
\hline
\end{tabular}

reported frequent use of miniscrews and $62.7 \%$ reported the use of miniscrews for the past $1-5$ years. Furthermore, $98.3 \%$ were completely or relatively satisfied with miniscrews, and $93.5 \%$ had recommended the use of miniscrews to their colleagues. Miniscrews had been most commonly placed for patients between 19 and 30 years $(71.3 \%)$. Miniscrews had been often

Table 2: Parameters related to miniscrew users

\begin{tabular}{|c|c|c|}
\hline Variables & Frequency & Percentage \\
\hline \multicolumn{3}{|l|}{ Duration of miniscrew usage (years) } \\
\hline $1<$ & 4 & 6.8 \\
\hline $1-5$ & 37 & 62.7 \\
\hline$>5$ & 18 & 30.5 \\
\hline \multicolumn{3}{|l|}{ Suggesting the use of miniscrews to colleagues } \\
\hline Yes & 58 & 93.5 \\
\hline No & 4 & 6.5 \\
\hline \multicolumn{3}{|l|}{ Use of miniscrew based on patients' age group (years) } \\
\hline$<12$ & 0 & 0 \\
\hline $12-18$ & 18 & 30.6 \\
\hline $19-30$ & 42 & 71.3 \\
\hline$>30$ & 9 & 15.3 \\
\hline All ages & 5 & 8.5 \\
\hline \multicolumn{3}{|l|}{ Operator who inserts the miniscrew } \\
\hline General dentist in another office & 0 & 0 \\
\hline Orthodontists themselves & 57 & 96.6 \\
\hline Oral surgeons or periodontists in another office & 2 & 3.4 \\
\hline General dentist in the same office & 0 & 0 \\
\hline \multicolumn{3}{|l|}{ Anesthesia of maxillary palatal plate } \\
\hline Topical & 12 & 20.7 \\
\hline Infiltration & 46 & 79.4 \\
\hline No anesthesia & 0 & 0 \\
\hline \multicolumn{3}{|l|}{ Anesthesia of maxillary buccal plate } \\
\hline Topical & 8 & 14.8 \\
\hline Infiltration & 46 & 85.2 \\
\hline No anesthesia & 0 & 0 \\
\hline \multicolumn{3}{|l|}{ Anesthesia of mandibular buccal plate } \\
\hline No anesthesia & 7 & 13.2 \\
\hline Topical & 9 & 17.0 \\
\hline Infiltration anesthesia & 0 & 0 \\
\hline Inferior alveolar nerve block & 37 & 69.8 \\
\hline
\end{tabular}

placed by the orthodontists themselves (96.6\%). Infiltration anesthesia was the most common anesthesia technique for the maxillary palatal $(79.4 \%)$ and maxillary buccal $(85.2 \%)$ plates. Inferior alveolar nerve block was the most commonly used technique of anesthesia for the mandibular buccal plate $(69.8 \%)$.

Table 3 presents other parameters related to miniscrew users. Type of tooth movement induced by the use of miniscrews was retraction in $86.4 \%$. Radiography was the most commonly used technique $(79 \%)$ to ensure no contact with the roots, and $66.2 \%$ recommended mouthwash for disinfection of the oral environment prior to miniscrew placement. Jeil was the most commonly used miniscrew brand ( $82.8 \%$ ), while the most commonly used miniscrew head type was the hooked type with a hole $(36.2 \%)$, slot $(36.2 \%)$, or both $(39.6 \%)$. The maximum length and diameter of miniscrews used were $8 \mathrm{~mm}(89.8 \%)$ and $1.6 \mathrm{~mm}(79.2 \%)$, respectively.

Table 3: Other factors related to miniscrew users

\begin{tabular}{|c|c|c|}
\hline Variables & Frequency & Percentage \\
\hline \multicolumn{3}{|l|}{ Type of tooth movement by use of miniscrews } \\
\hline Protraction & 32 & 54.2 \\
\hline Retraction & 51 & 86.4 \\
\hline Up righting & 14 & 23.7 \\
\hline Anterior intrusion & 27 & 45.8 \\
\hline Posterior intrusion & 34 & 57.6 \\
\hline Other & 3 & 5.1 \\
\hline \multicolumn{3}{|l|}{ Strategy adopted to prevent root contact } \\
\hline Angulation & 23 & 40.3 \\
\hline Pre-operative radiographic assessment & 45 & 79 \\
\hline Post-operative radiographic assessment & 13 & 22.8 \\
\hline Observation of bleeding, patient's pain sensation & 14 & 24.6 \\
\hline Use of surgical guide & 0 & 0 \\
\hline \multicolumn{3}{|l|}{ Hygiene measure practiced prior to miniscrew insertion } \\
\hline Use of mouthwash prior to miniscrew placement & 37 & 66.2 \\
\hline Use of mouthwash after miniscrew placement & 16 & 28.6 \\
\hline Use of mouthwash during miniscrew placement & 10 & 17.9 \\
\hline Antibiotic therapy prior to miniscrew placement & 2 & 3.6 \\
\hline Antibiotic therapy after miniscrew placement & 2 & 3.6 \\
\hline Other & 5 & 8.9 \\
\hline \multicolumn{3}{|l|}{ Miniscrew brand most commonly used } \\
\hline Ormco & 3 & 5.2 \\
\hline Protect & 2 & 3.4 \\
\hline Ao & 0 & 0 \\
\hline OsteoMed & 0 & 0 \\
\hline Jeil & 48 & 82.8 \\
\hline Ortholution & 0 & 0 \\
\hline Ortho organizer & 1 & 1.7 \\
\hline MIB & 1 & 1.7 \\
\hline Other & 3 & 1.7 \\
\hline \multicolumn{3}{|l|}{ Miniscrew length } \\
\hline $4 \mathrm{~mm}$ & 2 & 3.4 \\
\hline $6 \mathrm{~mm}$ & 21 & 35.6 \\
\hline $8 \mathrm{~mm}$ & 52 & 98.8 \\
\hline $10 \mathrm{~mm}$ & 31 & 52.6 \\
\hline $12 \mathrm{~mm}$ & 1 & 1.7 \\
\hline \multicolumn{3}{|l|}{ Miniscrew diameter } \\
\hline $1.2 \mathrm{~mm}$ & 3 & 5.1 \\
\hline $1.4 \mathrm{~mm}$ & 26 & 44.7 \\
\hline $1.6 \mathrm{~mm}$ & 46 & 79.2 \\
\hline $1.8 \mathrm{~mm}$ & 13 & 22.2 \\
\hline $2 \mathrm{~mm}$ & 10 & 17.1 \\
\hline \multicolumn{3}{|l|}{ Miniscrew head type } \\
\hline Plain with hook & 16 & 27.6 \\
\hline Hooked with slot & 21 & 36.2 \\
\hline Hooked with hole & 21 & 36.2 \\
\hline Hooked with hole and slot & 23 & 39.6 \\
\hline
\end{tabular}

Table 4 shows the parameters related to miniscrew non-users. As shown, lack of expertise $(33.3 \%)$ was the most common reason for not using miniscrews. High cost was the most common reason for no longer use of miniscrews (100\%). Furthermore, $42.9 \%$ of the participants discussed that they may use miniscrews in the future if they acquire adequate expertise in this respect through participation in hands-on workshops. 
Table 4: Parameters related to miniscrew non-users

\begin{tabular}{lll}
\hline Variables & Frequency & Percentage \\
\hline Reasons for no longer use of miniscrews & & \\
Losing a high number of miniscrews & 0 & 0 \\
High cost & 1 & 100.0 \\
Excessive daily management & 0 & 0 \\
Discouraging results & 0 & 0 \\
Patient complaints & 0 & 0 \\
Possibility of future use of miniscrews & 3 & 42.9 \\
$\quad$ Given that educational workshops are held & 1 & 14.3 \\
$\quad$ Given that their mechanical properties are improved & 1 & 14.3 \\
Given that their cost decreases & 2 & 28.6 \\
$\quad$ Under no condition & & \\
\hline
\end{tabular}

\section{Discussion}

This study assessed the frequency of using miniscrews by the Iranian orthodontists in 2020. The results showed that Iranian orthodontists were interested in using miniscrews, and $62.3 \%$ of the participants were familiar with miniscrews and were interested in using them. Male orthodontists were more interested in using miniscrews (60\%). A study conducted in France reported that the majority of orthodontists that reported the use of miniscrews were males $(51.85 \%)$, although their study population comprised $52 \%$ females and $48 \%$ males [7]. A study conducted in Germany also indicated that the majority of orthodontists who reported the use of miniscrews were males, although their study population comprised of $54 \%$ females. In their study, $61.29 \%$ of females who filled out the questionnaire reported the use of miniscrews, while this rate was $71.89 \%$ among males [9]. Meeran et al. [6] reported that only $43.7 \%$ of participants were interested in using miniscrews; this rate was lower than the corresponding values reported by other studies [7], [9], [10]. Another study reported the use of miniscrews by $91 \%$ of the participants. They reported that orthodontists attending the University of Washington commonly used miniscrews, while this rate was not so high in other parts of the United States [10]. The level of satisfaction of orthodontists with miniscrew placement was high such that $50.8 \%$ had complete and $47.5 \%$ had relative satisfaction with miniscrew placement in our study. The satisfaction level of French orthodontists with miniscrew treatment was $74.46 \%$ [7], while this rate was $70.75 \%$ in Germany [9] and $82 \%$ in Washington, United States [10]. The mean age of the majority of miniscrew users was 30-39 years in our study, accounting for about $50 \%$ of orthodontists. Those between 40 and 49 years ranked second (32.9\%), and orthodontists $>50$ years and $<30$ years had the least interest in use of miniscrews. In the study by Barthelemi and Beauval [7], 26.09\% of orthodontists $>50$ years believed that miniscrews could have a small role in the completion of treatment, and the majority of those using the miniscrews $(73.71 \%)$ were between 40 and 49 years.

Orthodontists with orthodontic practice experience $>5$ years comprised the majority of miniscrew users, accounting for $55.7 \%$ of our study population. In the study by Barthelemi and Beauval [7], two-thirds of miniscrew users had orthodontic practice experience $>5$ years. Since the use of miniscrew requires adequate expertise and experience in this respect, orthodontists with $>5$ years of work experience may be more successful in miniscrew placement. In this study, buccal placement of miniscrews (98.6\%) was the most common. Lingual surfaces were least commonly used for miniscrew placement. In the study by Barthelemi and Beauval [7], orthodontists with $\geq 5$ years of clinical experience used both maxillary and mandibular arches for miniscrew placement. However, the majority of participants preferred the maxilla for this purpose.

In this study, pre-operative radiography was the most important diagnostic modality employed to prevent root contact and for correct placement of miniscrews $(79 \%)$. This finding was in line with the results of Meeran et al., [6] who reported that panoramic radiography was the most commonly used diagnostic tool to guide the miniscrew (94.8\%) [6]. Buschang et al. [8] also reported radiography to be the best pre-operative diagnostic modality for miniscrew placement.

In our study, $96.6 \%$ of orthodontists stated that they would place the miniscrews by themselves, while in the study by Meeran et al., [6] general dentists placed miniscrews in $85.79 \%$ of the cases, and miniscrews were placed by oral surgeons and periodontists in $14.21 \%$ of the cases. Barthelemi and Beauval [7] reported that miniscrews were placed by orthodontists in $32 \%$ of the cases, while this rate was only $2 \%$ in the study by Bock and Ruf [9]; they discussed that miniscrew placement is a professional procedure and requires a high level of expertise. Of all 35\% reported that it is an easy procedure, $65 \%$ reported that it is relatively difficult, and $5 \%$ reported that it is a very difficult procedure [9].

Regarding the size of miniscrews, those with 8 $\mathrm{mm}$ height and $1.6 \mathrm{~mm}$ diameter were most commonly used. Another study reported that miniscrews with a diameter $<1.3 \mathrm{~mm}$ and length $<8 \mathrm{~mm}$ have a higher risk of fracture [10]. Miniscrew fracture causes inflammation and soft tissue growth, and is a major risk factor [11], [12]. In a study by Hyde et al. [10], miniscrews with 1.4-1.6 mm diameter and 8-10 $\mathrm{mm}$ length were most commonly used and yielded satisfactory results.

Poor expertise and skills with regard to miniscrew placement $(33.3 \%)$ and doubts regarding their advantages $(33.3 \%)$ were the main reasons expressed by the Iranian orthodontists to justify no use of miniscrews. Moreover, they believed that high cost and lengthy procedure are among other limitations that discourage orthodontists to use miniscrews. Furthermore, $100 \%$ of orthodontists who used to place miniscrews but were no longer practicing this technique mentioned the reason to be 
the high cost of miniscrews. In general, one important prerequisite for the use of miniscrews is to have adequate knowledge and expertise in this respect. No use of miniscrew by orthodontists in India was explained to be due to the absence of clinical practice and education in this respect $(67 \%)$, concerns regarding root damage or infection (54\%), reluctance of patients and not being able to persuade them to consent to this treatment $(29 \%)$, and not having the required instruments for miniscrew placement (14\%). Only $5 \%$ of orthodontists preferred conventional noninvasive treatments and $1 \%$ were concerned about the costs [6]. In France, the absence of experience and expertise in miniscrew placement was the main reason for female orthodontists' lack of interest in miniscrew placement [7].

Considering all the above, it appears that elimination of the expressed limitations can lead to widespread use of miniscrews by orthodontists in the future. Moreover, considering the role of patient satisfaction in use of a particular modality, future studies should focus on satisfaction level of patients with miniscrew insertion.

\section{Conclusion}

Considering the high efficacy of miniscrews for orthodontic treatment and high satisfaction level of Iranian orthodontists with miniscrews, their application is expected to rise by the Iranian orthodontists after eliminating the existing limitations against their widespread use.

\section{Authors' Contributions}

Study concept and design: Amin Golshah, Mohadese Feiz Rahnemoon, Zahra Bagheri, Nasim Karami. Acquisition of data: Zahra Bagheri, Mohadese Feiz Rahnemoon. Analysis and interpretation of data: Mohadese Feiz Rahnemoon. Drafting of the manuscript: Mohadese Feiz Rahnemoon, Zahra Bagheri, Nasim Karami. Critical revision of the manuscript for important intellectual content: Amin Golshah. Statistical analysis: Zahra Bagheri, Mohadese Feiz Rahnemoon. Administrative, technical, and material support: Amin Golshah. Study supervision: Amin Golshah.

\section{References}

1. Mohebbi S, Sheikhzadeh S, Bayanzadeh M, Batebizadeh A Oral impact on daily performance (OIDP) index in patients attending patients clinic at dentistry school of Tehran University of medical sciences. J Dent Med. 2012;25(2):135-41.

2. Janssen KI, Raghoebar GM, Vissink A, Sandham A. Skeletal anchorage in orthodontics-a review of various systems in animal and human studies. Int $\mathrm{J}$ Oral Maxillofac Implants. 2008;23(1):75-88.

PMid:18416415

3. Alkadhimi A, Al-Awadhi EA. Miniscrews for orthodontic anchorage: A review of available systems. J Orthod. 2018;45(2):102-14. https://doi.org/10.1080/14653125.2018.144 3873

PMid:29542406

4. Papadopoulos MA, Tarawneh F. The use of miniscrew implants for temporary skeletal anchorage in orthodontics: A comprehensive review. Oral Surg Oral Med Oral Pathol Oral Radiol Endod. 2007;103(5):e6-15. https://doi.org/10.1016/j. tripleo.2006.11.022

PMid: 17317235

5. Son S, Motoyoshi M, Uchida Y, Shimizu N. Comparative study of the primary stability of self-drilling and self-tapping orthodontic miniscrews. Am J Orthod Dentofacial Orthop. 2014;145(4):4805. https://doi.org/10.1016/j.ajodo.2013.12.020 PMid:24703286

6. Meeran NA, Venkatesh KG, Parveen MF. Current trends in miniscrew utilization among Indian orthodontists. J Orthod Sci. 2012;1(2):46-50. https://doi.org/10.4103/2278-0203.99762 PMid:24987625

7. Barthelemi S, Beauval $\mathrm{H}$. Prevalence of the use of anchorage miniscrews among French orthodontists. Int Orthod. 2015;13(4):436-61. https://doi.org/10.1016/j.ortho.2015.09.011 PMid:26507966

8. Buschang PH, Carrillo R, Ozenbaugh B, Rossouw PE. 2008 survey of AAO members on miniscrew usage. J Clin Orthod. 2008;42(9):513-8.

PMid: 18974458

9. Bock NC, Ruf S. Skeletal anchorage for everybody? A questionnaire study on frequency of use and clinical indications in daily practice. J Orofac Orthop. 2015;76(2):113-24, 126-8. https://doi.org/10.1007/s00056-014-0275-z

PMid:25744091

10. Hyde JD, King GJ, Greenlee GM, Spiekerman C, Huang GJ. Survey of orthodontists' attitudes and experiences regarding miniscrew implants. J Clin Orthod. 2010;44(8):481-6. PMid:21105585

11. Park HS, Lee SK, Kwon OW. Group distal movement of teeth using microscrew implant anchorage. Angle Orthod. 2005;75(4):602-9.

PMid:16097229

12. Viwattanatipa $\mathrm{N}$, Thanakitcharu S, Uttraravichien A, Pitiphat W. Survival analyses of surgical miniscrews as orthodontic anchorage. Am J Orthod Dentofacial Orthop. 2009;136(1):2936. https://doi.org/10.1016/j.ajodo.2007.06.018

PMid:19577145 